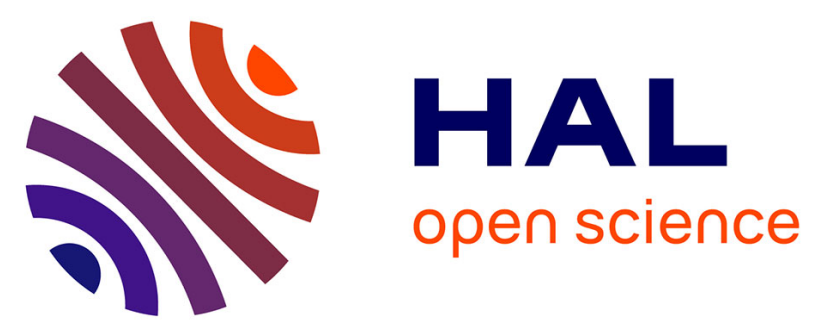

\title{
Performance Evaluation of Linear Precoding mmWave Multi-User MIMO Systems with NYUSIM Channel Simulator
}

Israa Khaled, Ammar El Falou, Charlotte Langlais, Bachar Elhassan, Michel Jezequel

\section{To cite this version:}

Israa Khaled, Ammar El Falou, Charlotte Langlais, Bachar Elhassan, Michel Jezequel. Performance Evaluation of Linear Precoding mmWave Multi-User MIMO Systems with NYUSIM Channel Simulator. MENACOMM, Nov 2019, Beirut, Lebanon. hal-02470155

\section{HAL Id: hal-02470155 \\ https://hal.science/hal-02470155}

Submitted on 7 Feb 2020

HAL is a multi-disciplinary open access archive for the deposit and dissemination of scientific research documents, whether they are published or not. The documents may come from teaching and research institutions in France or abroad, or from public or private research centers.
L'archive ouverte pluridisciplinaire HAL, est destinée au dépôt et à la diffusion de documents scientifiques de niveau recherche, publiés ou non, émanant des établissements d'enseignement et de recherche français ou étrangers, des laboratoires publics ou privés. 


\section{Performance Evaluation of Linear Precoding mmWave Multi-User MIMO Systems with NYUSIM Channel Simulator}

\author{
Israa Khaled \\ Lab-STICC, UMR CNRS 6285 \\ IMT Atlantique \\ Brest, France \\ israa.khaled@imt-atlantique.fr
}

\author{
Bachar ELHassan \\ Electrical and Electronics Department \\ Faculty of Engineering, Lebanese University \\ Tripoli, Lebanon \\ bachar_elhassan@ul.edu.lb
}

\author{
Ammar EL Falou \\ Electrical and Electronics Department \\ Faculty of Engineering, Lebanese University \\ Tripoli, Lebanon \\ ammar.elfalou@gmail.com
}

\author{
Charlotte Langlais \\ Lab-STICC, UMR CNRS 6285 \\ IMT Atlantique \\ Brest, France \\ charlotte.langlais@imt-atlantique.fr
}

\author{
Michel Jezequel \\ Lab-STICC, UMR CNRS 6285 \\ IMT Atlantique \\ Brest, France \\ michel.jezequel@imt-atlantique.fr
}

\begin{abstract}
In this paper, we evaluate the performance of the digital beamsteering (DBS) precoder in multi-user multiple-input multiple-output (MIMO) systems operating at mmWave frequencies. This evaluation is done using a 3-D mmWave statistical channel model developed based on outdoor extensive measurement in New York city. The NYUSIM channel simulator generates 3-D realistic statistical temporal and spatial parameters. The DBS performance is evaluated in terms of capacity achieved as a function of the number of users and compared with two classical precoders, namely, zero forcing ( $\mathrm{ZF}$ ) and conjugate beamforming (CB). The base station is equipped with three different antenna array configurations: uniform linear array, uniform square array and uniform rectangular array.

Simulation results show that DBS can be realistically considered as an attractive precoder for mmWave multi-user MIMO system with tens of antennas at the base station; trading-off performance, low implementation complexity and less overhead.

Index Terms-MU-MIMO, mmWave, linear precoding, antenna array architecture.
\end{abstract}

\section{INTRODUCTION}

The amount of available spectrum used by today's wireless communication systems is very limited compared to the future demands [1]. One promising solution is to move to millimeter wave (mmWave) bands, which offer a vast amount of unused spectrum, and thus achieves high data rates for future wireless communication systems [1], [2]. Moreover, multiuser multiple-input multiple-output (MU-MIMO) systems are essential technologies used to achieve high data rates and increase the diversity [3].

In order to assess the performance of mmWave MU-MIMO systems, the mmWave channel has to be modeled. For this purpose, several researchers have proposed their channel models such as: the 3rd Generation Partnership Project (3GPP) [4] and the New York University (NYU) wireless center referenced to as NYUSIM [5], [6]. The channel model proposed by 3GPP is mainly for bands below $6 \mathrm{GHz}$, with some adjustments to model the $6-100 \mathrm{GHz}$ band. In contrast, the statistical channel parameters of NYUSIM are generated based on extensive measurements in New York City at mmWave frequencies [5], [6]. The authors in [7], [8] compared the two channel models, and showed that NYUSIM offers more realistic simulation results compared to the $3 \mathrm{GPP}$ model. Based on their findings, we consider the NYUSIM model in our work.

In order to improve the spectral efficiency and reduce the inter-user interference, different linear precoding techniques can be used for MU-MIMO systems in the downlink [9][12]. In this paper, we are interested in the performance of digital beamsteering (DBS) precoder and its comparison with zero-forcing (ZF), conjugate beamforming (CB). The authors in [11], [12] studied both $\mathrm{CB}$ and ZF for MIMO systems and showed that these precoders are beneficial for large scale antennas. DBS is a less complex linear precoder, with respect to $\mathrm{ZF}$ and $\mathrm{CB}$, since it: i) requires only a partial knowledge of the channel state information (CSI) at the transmitter, mainly the angle of departure (AoD) of the LOS component of the channel, thus decreasing the channel overhead, ii) just uses digital phase shifters and iii) is a frequency non-selective precoder [13], [14]. DBS is then particularly suitable for multiuser transmissions in highly LOS environments. However, in [13], [14], DBS is evaluated at mmWave frequencies with a simple geometric channel model based on ray tracing, typically modeling 1 or 3 rays. A more realistic channel model, such as NYUSIM, has to be considered.

Furthermore, the presented works on $\mathrm{ZF}, \mathrm{CB}$ and DBS precoders do not consider the effect of the antenna array architecture on the achievable capacity of MU-MIMO systems. In [15], the impact of different antenna array architectures on MIMO channel properties is investigated. It is shown that these 
architectures have an important impact on the MIMO capacity and that the uniform linear array shows superiority over other geometries.

This paper revolves around the performance of DBS precoder in a realistic mmWave channel. Its performance is compared with two widely used precoders, namely ZF and CB. This evaluation is done in two LOS scenarios: urban microcell (UMi) and rural macrocell (RMa), based on the realistic 5G channel simulator NYUSIM. We consider three different types of antenna array architectures having a fixed inter-element spacing and the same number of antennas, namely, uniform linear array (ULA), uniform square array (USA) and uniform rectangular array (URA).

The rest of the paper is organized as follows. Section II introduces the system model, formulates the capacity of MUMIMO systems with linear precoding and describes the three different linear precoders ZF, CB and DBS. The mmWave channel model NYUSIM is presented in Section III. In Section IV, the simulation results are analyzed while Section V concludes the paper.

\section{System Model AND MiMO PReCoding}

We use the following notations: $\mathbf{X}$ is a matrix, $\mathbf{x}$ a vector, $x$ a scalar, $(.)^{T},(.)^{H}$ and $(.)^{-1}$ stand for the transpose, the Hermitian transpose, and matrix inverse, respectively.

\section{A. System Model}

We consider a downlink single-cell MU-MIMO system where the BS employs $M=M_{H} \times M_{V}$ transmit antennas, for serving $K$ single-antenna user equipments (UEs) with $K \leq M . M_{H}$ represents the number of horizontal antennas separated by $d_{H}$ inter-element spacing and $M_{V}$ represents the number of vertical antennas separated by $d_{V}$ inter-element spacing as shown in Fig. 1. Angles $(\theta, \phi)$ are the azimuth and the elevation angles, respectively. We assumed perfect OFDM transmission with $N_{F F T}$ subcarriers, where the channel $\mathbf{H}_{p} \in \mathbb{C}^{K \times M}$ is flat fading at each subcarrier $p$. The system is shown in Fig. 2, where $s_{p, k}$ represents the transmitted symbols intended for the $k$ th UE on subcarrier $p$. Since the mmWave channel is frequency-selective, the precoding system becomes frequencydependent. The BS applies a linear precoding $\mathbf{W}_{p} \in \mathbb{C}^{M \times K}$ before the transmission of signals through the antennas.

The precoded symbol vector $\mathbf{x}_{p} \in \mathbb{C}^{M \times 1}$ transmitted by the BS on subcarrier $p$ is given by:

$$
\mathbf{x}_{p}=\sqrt{\eta_{p}} \mathbf{W}_{p} \mathbf{s}_{p}
$$

with

$$
\eta_{p}=\frac{1}{\mathbb{E}\left[\operatorname{Tr}\left(\mathbf{W}_{p}{ }^{H} \mathbf{W}_{p}\right)\right]}
$$

where $\mathbf{s}_{p} \in \mathbb{C}^{K \times 1}$ denotes the original symbol vector for transmission on subcarrier $p$ with $\mathbb{E}\left[\operatorname{Tr}\left(\mathbf{s}_{p}{ }^{H} \mathbf{s}_{p}\right)\right]=1$ and $\eta_{p}$ is the normalization factor cancelling the precoding effect on channel power.
The received signal $\mathbf{r}_{p} \in \mathbb{C}^{K \times 1}$ on subcarrier $p$ is given by:

$$
\mathbf{r}_{p}=\mathbf{H}_{p} \mathbf{x}_{p}+\mathbf{n}_{p}=\sqrt{\eta_{p}} \mathbf{H}_{p} \mathbf{W}_{p} \mathbf{s}_{p}+\mathbf{n}_{p}
$$

where $\mathbf{n}_{p} \in \mathbb{C}^{K \times 1}$ is the additive white Gaussian noise (AWGN) vector, of zero-mean and $\sigma^{2}$-variance, affecting the received signal on subcarrier $p$.

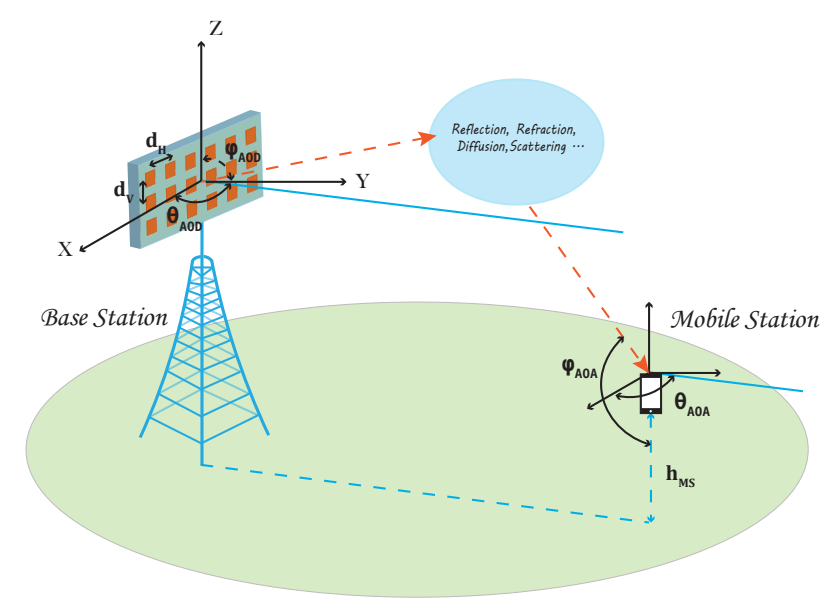

Fig. 1: Cell downlink MU-MIMO system

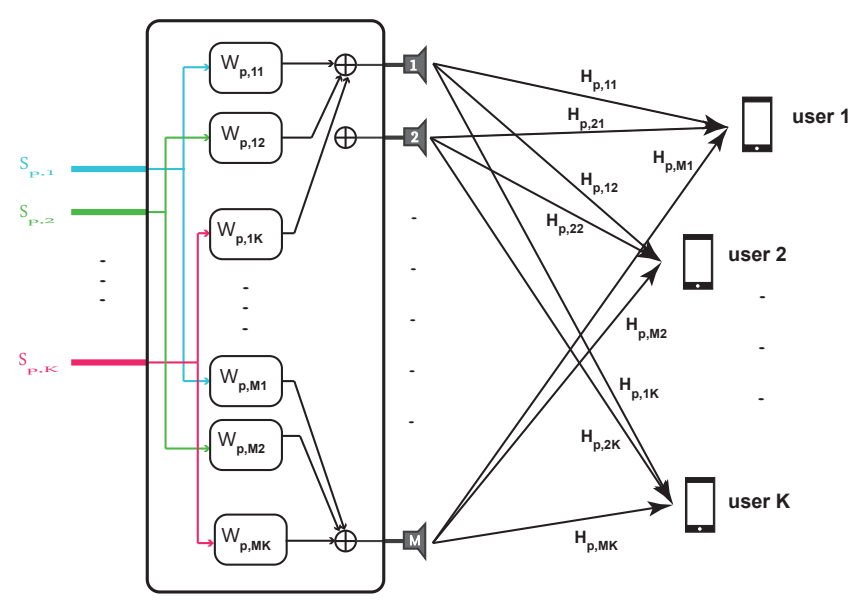

Fig. 2: System Model

\section{B. MU-MIMO Capacity}

The MU-MIMO capacity on subcarrier $p$ is defined as:

$$
C_{p}^{M U}=\log _{2} \operatorname{det}\left(\mathbf{I}_{K}+\frac{\mathbf{H}_{p} \boldsymbol{\Gamma}_{\boldsymbol{e}} \mathbf{H}_{p}^{H}}{\sigma^{2}}\right)
$$

Considering the precoder $\mathbf{W}_{p}$, the MU-MIMO capacity is expressed by:

$$
C_{p}^{M U}=\log _{2} \operatorname{det}\left(\mathbf{I}_{K}+\eta \frac{\mathbf{H}_{p} \mathbf{W}_{p} \boldsymbol{\Gamma}_{e} \mathbf{W}_{p}^{H} \mathbf{H}_{p}^{H}}{\sigma^{2}}\right)
$$


where $\Gamma_{e}=\mathbb{E}\left[\mathbf{s . \mathbf { s } ^ { H }}\right]$ is the covariance matrix of emitted signal, $\sigma^{2}$ is the noise power and $\mathbf{I}_{K}$ is a $K \times K$ identity matrix. The total capacity is given by:

$$
C_{M U}=\frac{1}{N_{F F T}} \sum_{p=1}^{N_{F F T}} C_{p}^{M U}
$$

\section{MIMO Linear Precoding}

\section{1) Zero Forcing $(\mathbf{Z F})$}

With a full CSI at the transmitter, ZF splits the multiuser channel into multiple independent subchannels maximizing the signal to interference ratio. The $\mathrm{ZF}$ precoding matrix is given by [10]:

$$
\mathbf{W}_{p}{ }^{Z F}=\mathbf{H}_{p}{ }^{H}\left(\mathbf{H}_{p} \mathbf{H}_{p}{ }^{H}\right)^{-1}
$$

The implementation of matrix inversion makes $\mathrm{ZF}$ more complex and limits its performance. Thus, the reciprocal condition number of $\mathbf{H}_{p} \mathbf{H}_{p}{ }^{H}$ is calculated in section IV to determine its effect into $\mathrm{ZF}$ performance.

\section{2) Conjugate Beamforming (CB)}

$\mathrm{CB}$, known as matched filter, maximizes the signal to noise ratio using full $\mathrm{CSI}$ at the transmitter. The $\mathrm{CB}$ precoder is given by [10]:

$$
\mathbf{W}_{p}{ }^{C B}=\mathbf{H}_{p}{ }^{H}
$$

\section{3) Digital BeamSteering (DBS)}

DBS is an angular based precoder characterized by low implementation complexity and low overhead, since it only relies on the user direction. This direction is determined by the AoD of the LOS component and is perfectly known thanks to NYUSIM. DBS forms and steers a beam toward the intended user using digital phase shifters [13]. The DBS precoder is based on the steering matrix A corresponding to the LOS path:

$$
\mathbf{W}_{p}^{D B S}=\mathbf{A}^{H}
$$

with

$$
\mathbf{A}=\left[\begin{array}{llll}
\mathbf{a}_{1}, & \mathbf{a}_{2}, & \cdots & , \mathbf{a}_{K}
\end{array}\right]^{T}
$$

where $\mathbf{a}_{k}$ is the steering vector at $\operatorname{AoD}\left(\theta_{k}, \phi_{k}\right)$ corresponding to user $k$ [14]:

$$
\begin{aligned}
& \mathbf{a}_{k}=\left[1, \ldots, e^{j\left(M_{H}-1\right) 2 \pi / \lambda d_{H} \cos \left(\theta_{k}\right) \cos \left(\phi_{k}\right)}, \ldots,\right. \\
& \left.e^{j 2 \pi / \lambda\left(\left(M_{H}-1\right) d_{H} \cos \left(\theta_{k}\right) \cos \left(\phi_{k}\right)+\left(M_{V}-1\right) d_{V} \sin \left(\phi_{k}\right)\right)}\right]^{T}
\end{aligned}
$$

\section{NYUSIM MMWAVE CHANNEL MODEL}

Using the time cluster spatial lobe (TCSL) approach, NYU wireless center developed a 3-D mmWave statistical spatial channel model (SSCM) based on extensive measurements in diverse outdoor environments such as rural macrocell (RMa), urban macrocell (UMa) and urban microcell (UMi), at multiple mmWave frequencies in New York City [5]-[8]. NYUSIM is an open source channel model simulator describing the
SSCM model. In order to model the mmWave channel, the SSCM generates the 3-D angular power spectra and the omnidirectional/directional channel impulse responses (CIRs) in both time and space domains [6].

The omnidirectional CIR $\mathrm{h}_{\mathrm{omni}}(t, \overrightarrow{\boldsymbol{\Theta}}, \overrightarrow{\boldsymbol{\Phi}})$ is defined by:

$\mathrm{h}_{\mathrm{omni}}(t, \overrightarrow{\boldsymbol{\Theta}}, \overrightarrow{\boldsymbol{\Phi}})=\sum_{n=1}^{\text {nTap }} P_{n} e^{j \varphi_{n}} \delta\left(t-t_{n}\right) \delta\left(\overrightarrow{\boldsymbol{\Theta}}-\overrightarrow{\boldsymbol{\Theta}}_{n}\right) \delta\left(\overrightarrow{\boldsymbol{\Phi}}-\overrightarrow{\boldsymbol{\Phi}}_{n}\right)$

where $t$ denotes the absolute propagation time, $\overrightarrow{\boldsymbol{\Theta}}=(\theta, \phi)_{\mathrm{TX}}$, $\overrightarrow{\mathbf{\Phi}}=(\theta, \phi)_{\mathrm{RX}}$ are the vectors of azimuth and elevation AoDs and angle of arrivals (AoAs), respectively and nTap denotes the number of subpaths (SPs). $P_{n}, \varphi_{n}, t_{n}$, are the amplitude, phase and propagation time delays, respectively of the $n$th SP. $\overrightarrow{\boldsymbol{\Theta}}_{n}$ et $\overrightarrow{\boldsymbol{\Phi}}_{n}$ are its azimuth/elevation AoDs and azimuth/elevation AoAs, respectively. Note that each SP belongs to a time cluster (TC) and a spatial lobe (SL). The MIMO channel model is obtained by considering the paths between antennas as in [12].

We have plotted the SPs for both RMa and UMi. Fig. 3a shows that several SPs belonging to different SLs exist in UMi while only one or two SPs belonging to the same SL exist in RMa as depicted in Fig. 3b.

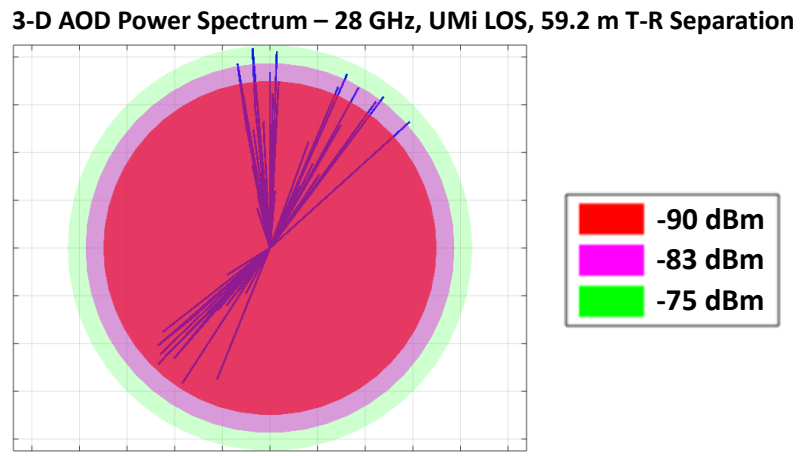

(a) UMi

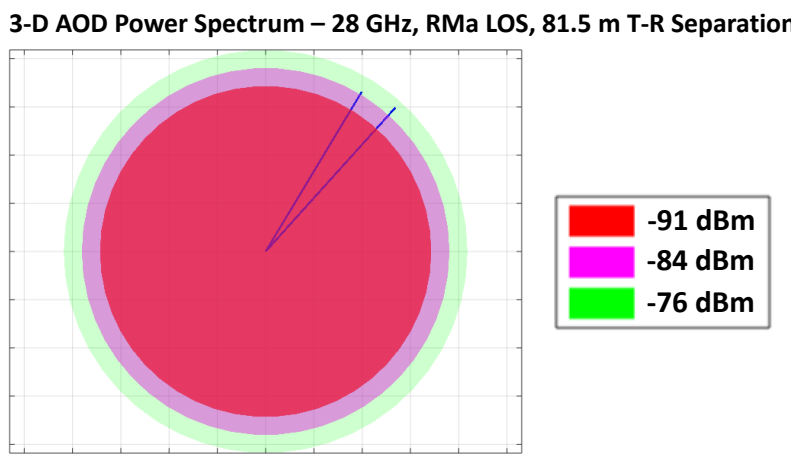

(b) RMa

Fig. 3: Simulated AoD Power Spectrum.

\section{Simulation Results}

In this section, we numerically compare the performance of $\mathrm{CB}, \mathrm{ZF}$ and DBS in terms of capacity achieved as a function of the number of served users in two different LOS 
environments: RMa and UMi. All temporal and spatial parameters of mmWave channel were generated using NYUSIM simulator. The MU-MIMO simulated system operates at 28 $\mathrm{GHz}$ and with a radio frequency bandwidth of $800 \mathrm{MHz}$. The BS employs three different antenna array architectures: ULA, URA and USA having $M=16$ antennas. The inter-element spacing is set at $\lambda / 2 . M_{V}=16$ and $M_{H}=1$ for ULA, $M_{V}=M_{H}=4$ for USA, $M_{V}=2$ and $M_{H}=8$ for URA. The distance between the transmitter and the receiver varies uniformly between 10 and $100 \mathrm{~m}$. The total transmit power is assumed to be equal to $30 \mathrm{dBm}$ and the noise power equals to $-85 \mathrm{dBm}$ corresponding to typical receiver sensitivity. We take $N_{F F T}=1024$ subcarriers. In this evaluation, we assume a perfect CSI for CB and ZF precoders and a perfect knowledge of the LOS component angle for DBS precoder.

\section{A. UMi scenario}

Fig. 4 plots the capacity, achieved by various precoding in UMi scenario. For CB and ZF, ULA provides the best performance followed by URA then USA. For DBS, ULA outperforms the others when the number of users is close to the number of BS antennas, and both URA and USA outperform the ULA when less users are served simultaneously. Indeed for DBS, the linear array allows a great discrimination of users in azimuth and no discrimination in elevation, while the rectangular array allows users' discrimination in both azimuth and elevation. When the number of served users increases, more users will be close together and thus the discrimination in azimuth is more efficient. This explains the superiority of ULA for this case. In contrast, when less users are served simultaneously, the users will be far from each other and thus the discrimination in both azimuth and elevation is the most efficient explaining the superiority of the URA and USA with respect to ULA.

\section{B. RMa scenario}

Fig. 5 plots the capacity, achieved by various precoding in RMa scenario. For CB, ULA has the superiority over other geometries. In contrast, using ZF, URA outperforms the others. For DBS, both URA and USA outperform ULA for less served users in the cell, while URA outperforms the others for more users.

\section{Results discussion}

By comparing Fig. 4 and Fig. 5, the capacity is greater in UMi than in RMa. This difference comes from a greater number of SPs in the case of UMi, and thus a better spatial and frequency diversities, as illustrated in Fig. 3a and $3 b$.

Furthermore, both CB and DBS supports 16 users, while the performance of $\mathrm{ZF}$ is limited by the number of served users. In both UMi and RMa, the maximum number of users supported by the ZF system is the same, i.e, 13 for USA, 15 for URA and 16 for ULA. Figures $6 \mathrm{a}, 6 \mathrm{~b}$ and $6 \mathrm{c}$ explain these values. Indeed, these figures plot the distribution of the base-10 logarithm of the reciprocal condition number (Rcond) of $\mathbf{H}_{p} \mathbf{H}_{p}{ }^{H}$ or $\log _{10}\left(\operatorname{Rcond}\left(\mathbf{H}_{p} \mathbf{H}_{p}{ }^{H}\right)\right)$ in

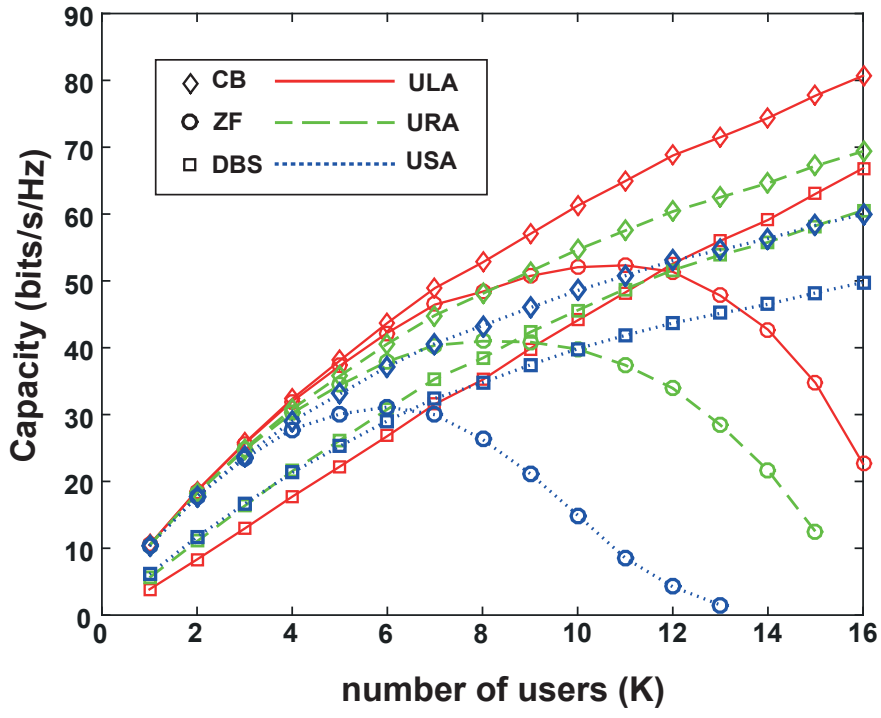

Fig. 4: Capacity achieved using ZF, CB and DBS for a $16 \times 1$ MIMO system at $28 \mathrm{GHz}$ with ULA (red line), URA (green dashed line) and USA (blue dotted line) at the transmitter in LOS UMi scenario. The results are averaged over 1000 runs.

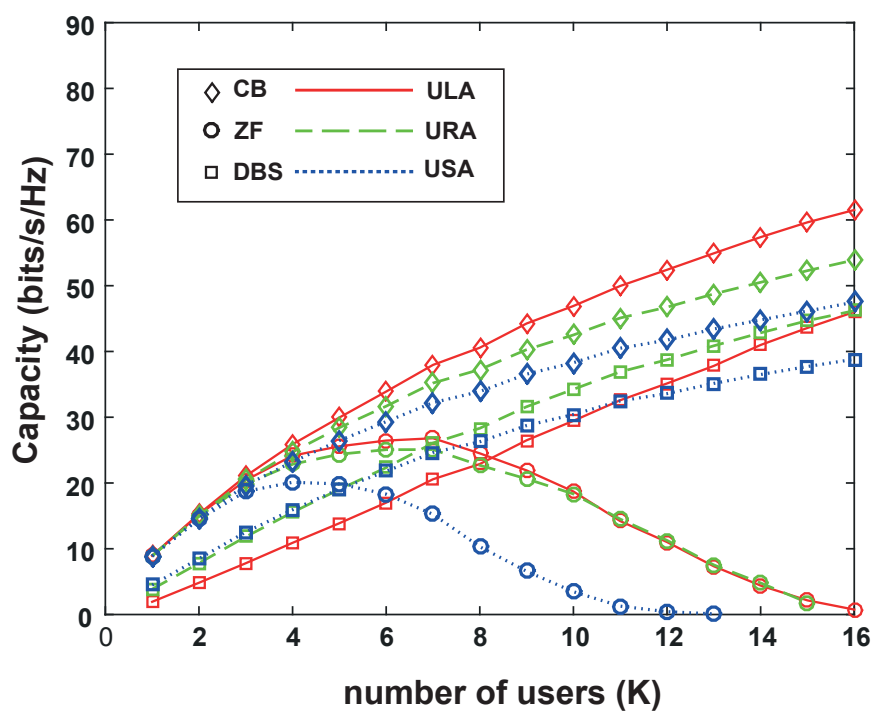

Fig. 5: Capacity achieved using ZF, CB and DBS for a $16 \times 1$ MIMO system at $28 \mathrm{GHz}$ with ULA (red line), URA (green dashed line) and USA (blue dotted line) at the transmitter in LOS RMa scenario. The results are averaged over 1000 runs.

UMi scenario. Indeed, when Rcond is near to 1 or $0 \mathrm{~dB}$ the matrix $\mathbf{H}_{p} \mathbf{H}_{p}{ }^{H}$ is well-conditioned, while it is ill-conditioned when Rcond is very small. The figures show that for URA, $\log _{10}\left(\operatorname{Rcond}\left(\mathbf{H}_{p} \mathbf{H}_{p}{ }^{H}\right)\right)$ takes values around -18 when the number of users is 16 , this means that for 16 users the $\mathrm{ZF}$ precoding involving a matrix inversion is ill-conditioned. Thus, the maximum number of supported users by the ZF precoder $\left(K_{\max }\right)$ is equal to 15 for URA. Similarly, in USA, $K_{\max }$ is equal to 12 . The ULA supports 16 users as its respected values 


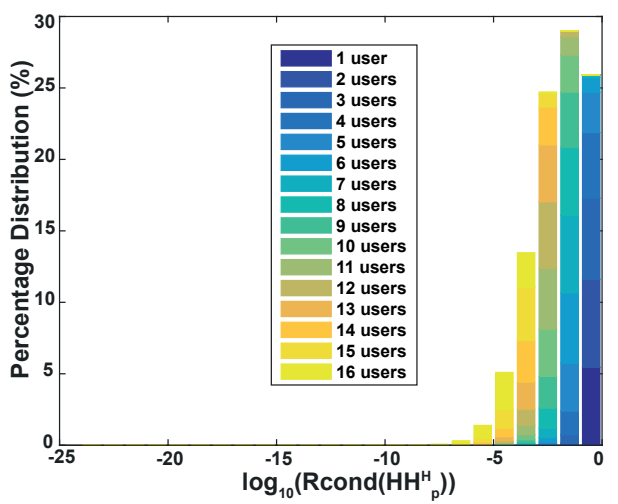

(a) ULA

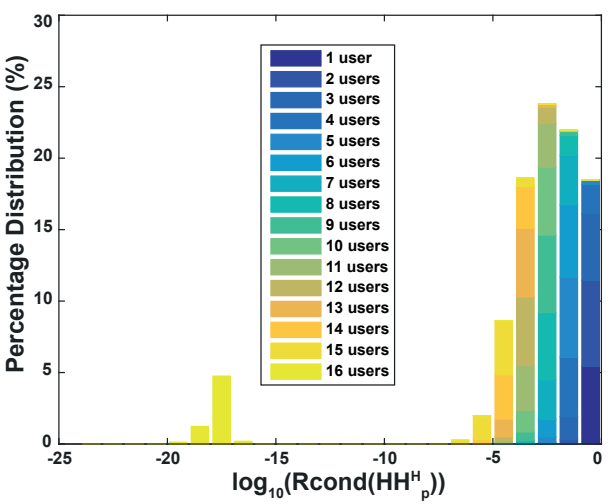

(b) URA

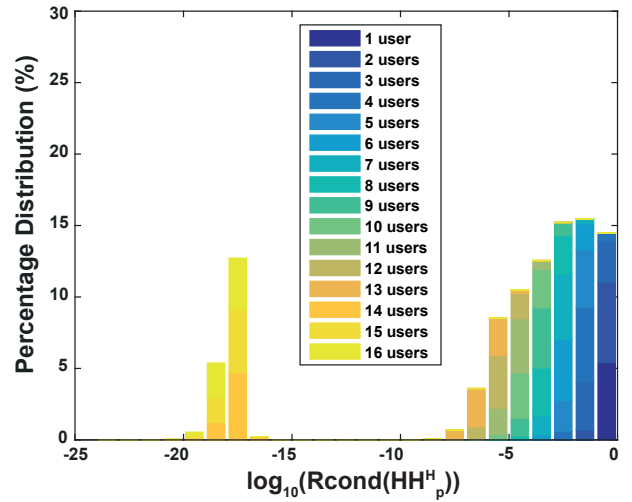

(c) USA

Fig. 6: Percentage distribution of $\log _{10}\left(\operatorname{Rcond}\left(\mathbf{H}_{p} \mathbf{H}_{p}{ }^{H}\right)\right)$ in UMi scenario for ULA, URA and USA.

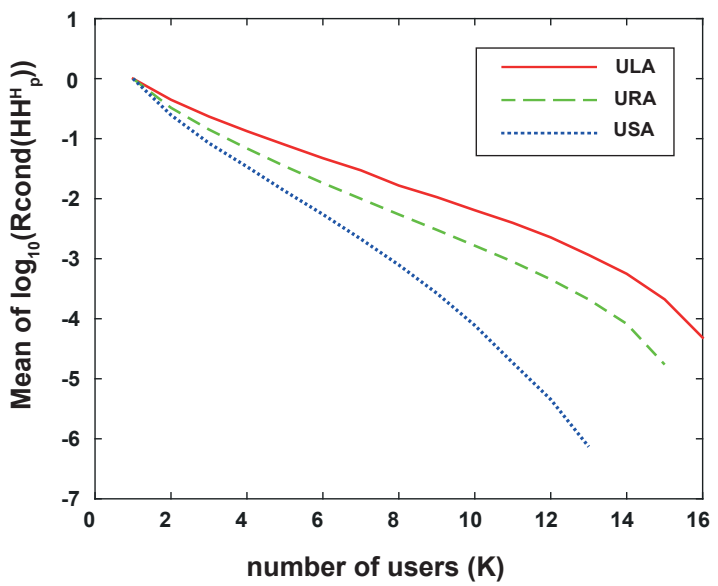

(a) UMi

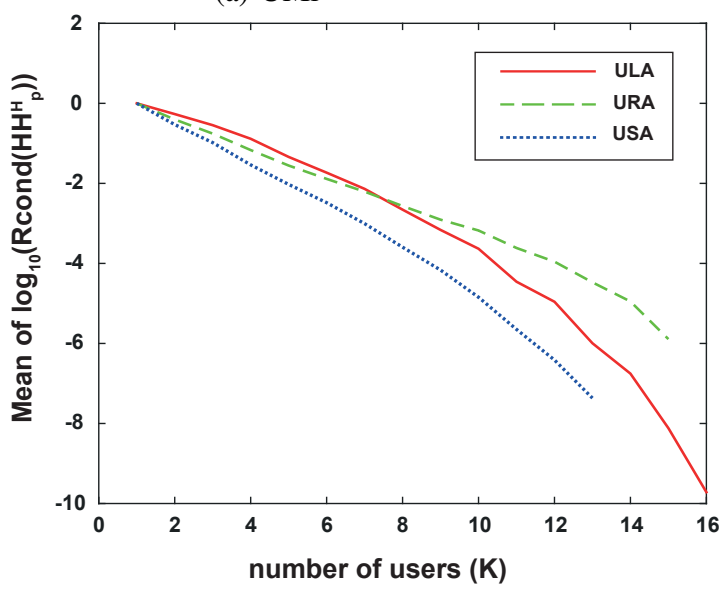

(b) RMa

Fig. 7: Average of $\log _{10}\left(\operatorname{Rcond}\left(\mathbf{H}_{p} \mathbf{H}_{p}{ }^{H}\right)\right)$ for ULA, URA and USA in both UMi and RMa scenarios. are always greater than -10 . Moreover, the average value of $\log _{10}\left(\operatorname{Rcond}\left(\mathbf{H}_{p} \mathbf{H}_{p}{ }^{H}\right)\right)$ gives an insight on the obtained capacities of ZF for RMa and UMi. Fig. 7a shows that the hierarchy of this average value matches the one of ZF capacity in UMi scenario. In RMa scenario, Fig. 7b provides the similar conclusion.

Finally, CB always provides the best performance, and the maximum capacity difference between $\mathrm{CB}$ and DBS is about $10-12$ bits $/ \mathrm{s} / \mathrm{Hz}$ when 16 users are served simultaneously, whereas $\mathrm{ZF}$ precoder shows disastrous performance. This behavior in overloaded scenario is already known. However, choosing this kind of overloaded scenario is of great interest when taking into account the complexity brought by the increasing number of antennas and RF chains in mmWave scenario. DBS is a promising precoder as it is less complex than $\mathrm{CB}$ and $\mathrm{ZF}$ with a low overhead. It can be a good trade-off between low implementation complexity and capacity performance for multi-user transmission in mmWave systems, since the channel at mmWave is expected to be a highly LOS environment. In our work, we have shown that DBS maintains its performance even in a realistic channel and not only in a simple 3 rays environment as in previous works.

\section{Conclusion}

In this paper, we evaluate the performance of DBS precoder in mmWave MU-MIMO system using a realistic channel model, called NYUSIM. We have shown that DBS is wellsuited for mmWave multi-user transmission systems in highly LOS environment. Moreover, it is a promising fully digital precoder, characterized by its low implementation complexity and channel overhead with respect to $\mathrm{ZF}$ and $\mathrm{CB}$ precoders.

The design of an estimator for the AoD of the LOS SP, and the evaluation of the DBS robustness against estimation errors in terms of AoDs are important perspectives for this work towards a more realistic scenario.

\section{ACKNOWLEDGMENT}

This work has been funded by IMT Atlantique, Lebanese University and the AZM association. 
The authors would like to thank Shihao Ju, Shu Sun and Theodore S. Rappaport of NYU Wireless, and New York University for providing open source simulator referenced as NYUSIM.

\section{REFERENCES}

[1] T. S. Rappaport, S. Sun, R. Mayzus, H. Zhao, Y. Azar, K. Wang, G. N. Wong, J. K. Schulz, M. Samimi, and F. Gutierrez Jr, "Millimeter wave mobile communications for 5G cellular: It will work!" IEEE access, vol. 1, no. 1, pp. 335-349, 2013.

[2] Z. Pi and F. Khan, "An introduction to millimeter-wave mobile broadband systems," IEEE Commun. Mag., vol. 49, no. 6, 2011.

[3] C. B. Peel, Q. H. Spencer, A. L. Swindlehurst, and M. Haardt, "An introduction to the multi-user mimo downlink," IEEE Commun. Mag., vol. 61, 2004.

[4] 3GPP. (2017, Sep.) Technical specification group radio access network; study on channel model for frequencies from 0.5 to $100 \mathrm{ghz}$ (release 14). [Online]. Available: http://www.3gpp.org/DynaReport/38901.html

[5] T. S. Rappaport, G. R. MacCartney, M. K. Samimi, and S. Sun, "Wideband millimeter-wave propagation measurements and channel models for future wireless communication system design," IEEE Trans. Commun., vol. 63, no. 9, pp. 3029-3056, 2015.

[6] M. K. Samimi and T. S. Rappaport, "3-D millimeter-wave statistical channel model for 5G wireless system design," IEEE Trans. Microw. Theory Tech., vol. 64, no. 7, pp. 2207-2225, 2016.

[7] T. S. Rappaport, S. Sun, and M. Shafi, "Investigation and comparison of 3 GPP and NYUSIM channel models for $5 \mathrm{G}$ wireless communications," in Proc. IEEE Vehicular Technology Conference (VTC), 2017.

[8] S. Sun, T. S. Rappaport, M. Shafi, P. Tang, J. Zhang, and P. J. Smith, "Propagation models and performance evaluation for $5 \mathrm{G}$ millimeterwave bands," IEEE Trans. Veh. Technol., vol. 67, no. 9, pp. 8422-8439, 2018.

[9] M. Vu and A. Paulraj, "MIMO wireless linear precoding," IEEE Signal Process. Mag., vol. 24, no. 5, pp. 86-105, 2007.

[10] N. Fatema, G. Hua, Y. Xiang, D. Peng, and I. Natgunanathan, "Massive MIMO linear precoding: A survey," IEEE Syst. J., no. 99, pp. 1-12, 2017.

[11] H. Yang and T. L. Marzetta, "Performance of conjugate and zeroforcing beamforming in large-scale antenna systems," IEEE J. Sel. Areas Commun., vol. 31, no. 2, pp. 172-179, 2013.

[12] M. El Hassan, A. El Falou, and C. Langlais, "Performance assessment of linear precoding for multi-user massive MIMO systems on a realistic 5G mmwave channel," in Proc. IEEE Middle East and North Africa Communications Conference (MENACOMM), Apr. 2018.

[13] A. Rozé, M. Hélard, M. Crussière, and C. Langlais, "Millimeter-wave digital beamsteering in highly line-of-sight environments for massive MIMO systems," in Wireless World Research Forum Meeting, vol. 35, 2015.

[14] M. Shehata, M. Hélard, M. Crussière, A. Rozé, and C. Langlais, "Angular based beamforming and power allocation framework in a multi-user millimeter-wave massive MIMO system," in Proc. IEEE Vehicular Technology Conference (VTC), Jun. 2018.

[15] A. A. Abouda, H. M. El-Sallabi, and S. Häggman, "Effect of antenna array geometry and ula azimuthal orientation on MIMO channel properties in urban city street grid," Progress In Electromagnetics Research, vol. 64, pp. 257-278, 2006. 